\title{
OPTIMAL CLAIM DECISIONS FOR A BONUS-MALUS SYSTEM: A CONTINUOUS APPROACH*
}

\author{
NeLson De PrIL
}

\section{INTRODUCTION}

For the premium calculation the insurer will split up his collectivity of risks into risk groups which are homogeneous with respect to some directly observable risk factors. All risks of such a risk group will be charged the same base premium. But it is clear that by such an a priori classification not all determined factors can be taken into consideration, so that there will still remain accident proneness differentials within a risk group. Since these differentials will be reflected in the course of time by the claim experience of each risk, the insurer can come to a fair tarification by adjusting, each period, the base premium according to the individual claim experience of the risk. Such a system in which earlier neglected risk factors are taken into account a posteriori is an individual experience rating system. Our main interest goes to the following side-effect of experience rating: since an unfavourable claim experience results in a premium increase, an experience rated policyholder is stimulated to self-insure small damages. This phenomenon is well know in connection with bonus-malus systems in motor-car insurance, which explains why it is called "bonus-hunger".

In the present paper a continuous time model for the bonus-malus system is set up which takes into account this hunger for bonus. An insured causing an accident will decide according to a certain decision rule whether to file a claim with his insurance company. The relevant information that he needs to make this decision is: his current risk class, the number of claims he has already filed during that period and the moment at which the decision is to be made. The decision of an insured causing at time $t$ of period $n$ an accident which amounts to $L$, can thus be thought of as being based on a decision rule of the following general form

$$
L-L_{n}(i, k, t)\left\{\begin{array}{l}
>\text { o claim } \\
\leqslant \text { o do not claim }
\end{array}\right.
$$

with $L_{n}(i, k, t)$ the amount that the actual accident must exceed in order to justify the filing of a claim, if the insured is at time $t$ of period $n$ in risk class $i$ and has already filed $k$ claims. The determination of the critical claim size

* An earlier version of this paper was presented at the $14^{\text {th }}$ ASTIN Colloquium, Taormina, October 1978. 
$L_{n}(i, k, t)$ should be made on economic grounds. Typical non-optimal critical claim sizes are some positive constant or the first year difference between the insurance premiums for filing and not filing a claim. The optimal value of $L_{n}(i, k, t)$ is clearly the one that minimizes the discounted expectation of the total future cost (premiums and self-defrayed claims) of the policyholder.

The problem of determining the optimal critical claim size was tackled in several papers under different restricting assumptions. In some of them an experience rating method is considered which avoids difficulties appearing by the general bonus-malus system; e.g. in DE LEVE and WEEDA (1968) and WEEDA (1975) a pure bonus system is considered in which the policyholder is classified according to the number of claimfree years since the last claim, so that the decision to file or not to file a claim exists only if no claim has been made during the same period. In other papers the general model in which the decision is to be taken is not satisfying; e.g. in the model of LemaIRE (1976-77) a policyholder remains always insured which leads to a critical claim size that is independent of the period in which the accident takes place. Finally there are papers in which restrictions are made on the form of the decision rule itself; e.g. MARTIN-LöF (1973) supposes that the decision whether to file a claim has to be made at the end of the insurance period.

The most general approach to this problem was given by HaEHLING voN LANZENauer (1974), who considers a discrete time model and determines the optimal critical claim size by dynamic programming. However his formulation seems contradictory since he takes on the one side that the number of accidents is Poisson distributed and on the other side that in a short-but finite-time interval no more than one accident can occur. With a continuous model this problem will be avoided.

\section{DESCRIPTION OF THE MODEL}

We consider a risk group in which the accident proneness of a risk is represented by a risk parameter $\lambda$ which is constant in time. We assume that the risks are independent so that we can restrict ourselves to the discussion of a single risk. We take a risk $\lambda$ and assume that the number of accidents in each time interval of length $t$ is Poisson distributed with mean $\lambda t$. Further we introduce the following notations:

$f_{n}(l)$ density function of a claim amount in period $n$.

$F_{n}(l)$ the corresponding distribution function.

$w_{n} \quad$ probability that the risk remains insured for the period $n$ if it was insured for the period $n-1$. For the first period we have $w_{1}=1$, and if we introduce a last period $N$ after which each risk has left the system with certainty, we have $w_{N+1}=0$.

$\delta \quad$ force of interest. 
We assume that the tarification in the risk group is based on a bonusmalus system that is determined in the following way.

- The length of an insurance period is 1.

- The number of classes in $J$.

- The class in which a risk is placed during the first period in the class $s$.

- The premium that a risk of class $j$ has to pay at the beginning of period $n$ to be insured for this period is $b_{n}(j)(j=1, \ldots, J ; n=1, \ldots, N)$.

- The transition rules are given in the form of probabilities $t_{i j}(k)(i, j=$ $1, \ldots, J ; k=0,1, \ldots)$ where $t_{i j}(k)=1$ if a risk of class $i$ moves to class $j$ when $k$ claims were filed in the past period, and $t_{i j}(k)=0$ if such a risk goes to a class different from $j$. In order that the transition rules be complete and free of contradictions we must have: for each $(i, k)$ there is one and only one $j$ so that $t_{i j}(k)=1$.

By convention the classes are numbered so that the highest premium corresponds to the class $J$. Then we have in a reasonable system that $t_{i j}(k)=$ $t_{i j^{\prime}}\left(k^{\prime}\right)=1$, with $k>k^{\prime}$, implies $j \geqslant j^{\prime}$. By definition we call characteristic claim number of a class $i$ the minimal number of filed claims that makes that a risk of this class will go to the highest class $J$ for the following period. The characteristic claim numbers $K_{i}$ are thus determined by

$$
t_{i J}(k)= \begin{cases}0 & \text { for } k=0, \ldots, K_{i}-1 \\ 1 & \text { for } k=K_{i}, K_{i}+1, \ldots\end{cases}
$$

3. THE EXPECTATION OF THE TOTAL COST FOR THE POLICYHOLDER

We consider a risk $\lambda$ who decides whether to file a claim according to a given decision rule of the form (1), where $L_{n}(i, k, t)$ is continuous in $0 \leqslant t<1$. Let $A_{n}(i, k, t)$ represent the discounted expectation of all future cost (premiums and self-defrayed claims) if the risk is currently at time $t$ of period $n$, belongs to risk class $i$, and has already filed $k$ claims that period. According to the assumption that the number of claims is Poisson distributed, we have in a (short) time interval of length $\Delta t$ that

$$
\begin{aligned}
& A_{n}(i, k, t)=(1-\lambda \Delta t) e^{-\delta \Delta t} A_{n}(i, k, t+\Delta t) \\
& +\lambda \Delta t F_{n}\left[L_{n}(i, k, t+\S)\right]\left\{e^{-\delta \S} \int_{0}^{L_{n}(i, k, t+\xi)} l f_{n}\left[l \mid l \leqslant L_{n}(i, k, t+\S)\right] d l\right. \\
& \left.+e^{-\delta \Delta t} A_{n}(i, k, t+\Delta t)\right\}+\lambda \Delta t\left\{1-F_{n}\left[L_{n}\left(i, k, t+\S^{\prime}\right)\right]\right\} e^{-\delta \Delta t} A_{n}(i, k+1, t+\Delta t) \\
& +o(\Delta t)
\end{aligned}
$$

where $0<\S, \S^{\prime}<\Delta t$. Hereby $o(\Delta t)$ denotes a function $f(\Delta t)$ for which lim $\frac{f(\Delta t)}{\Delta t}=0$. 
Dividing by $\Delta t$, we have

$$
\begin{aligned}
& \frac{A_{n}(i, k, t+\Delta t)-A_{n}(i, k, t)}{\Delta t}=\frac{1}{\Delta t}[\delta \Delta t+o(\Delta t)] A_{n}(i, k, t+\Delta t) \\
& +\lambda\left\{1-F_{n}\left[L_{n}(i, k, t+\S)\right]\right\} e^{-\delta \Delta t} A_{n}(i, k, t+\Delta t) \\
& -\lambda\left\{1-F_{n}\left[L_{n}\left(i, k, t+\S^{\prime}\right)\right]\right\} e^{-\delta \Delta t} A_{n}(i, k+1, t+\Delta t) \\
& -\lambda e^{-\delta \S} \int_{0}^{L_{n}(i, k, t+\S)} l f_{n}(l) d l-\frac{o(\Delta t)}{\Delta t}
\end{aligned}
$$

and by letting $\Delta t \rightarrow 0$, we obtain

$$
\begin{aligned}
\frac{d A_{n}(i, k, t)}{d t} & =\delta A_{n}(i, k, t)+\lambda\left\{1-F_{n}\left[L_{n}(i, k, t)\right]\right\}\left[A_{n}(i, k, t)-\right. \\
& \left.-A_{n}(i, k+1, t)\right]-\lambda \int_{0}^{L_{n}(i, k, t)} l f_{n}(l) d l
\end{aligned}
$$

Boundary conditions are found by considering the left-hand limit of $A_{n}(i, k, t)$ at $t=1$.

(4a) $\quad \int A_{n}(i, k, 1)=w_{n+1} \sum_{j=1}^{J}\left[b_{n+1}(j)+A_{n+1}(j, 0,0)\right] t_{i j}(k)$ if $n=1, \ldots, N-1$

(4b) $\quad\left(A_{N}(i, k, 1)=\mathrm{o}\right.$

In (4a) we have taken into account the premium for the period $n+1$ of the unique class $j$ determined by the class $i$ and the number of claims $k$ filed during period $n$. By means of the equations (3) and (4) every $A_{n}(i, k, t)$ can be determined recursively starting with $A_{N}(i, k, 1)=0$ for each $(i, k)$. The recurrence differential equation (3) determines the evolution of $A_{n}(i, k, t)$ through period $n$ and the formula (4a) gives the relation between the $A_{n}(i, k, t)$ for consecutive periods.

\section{THE OPTIMAL CRITICAL CLAIM SIZE}

A risk causing at time $t$ of period $n$ an accident which amounts to $L$ has the disposal of two strategies. When he does not file a claim the present value at the moment of the accident of the expectation of his total cost is $L+$ $A_{n}(i, k, t)$, where $i$ is his current risk class and $k$ is the number of claims he has already filed that period. When he files a claim the expectation of his total cost is $A_{n}(i, k+1, t)$. By definition the risk will make an optimal claim decision if the expectation of his total cost is minimized as a result of his decision making. The optimal critical claim size is thus

$$
L_{n}(i, k, t)=A_{n}(i, k+1, t)-A_{n}(i, k, t)
$$


According to (3), derivation gives

$$
\begin{aligned}
\frac{d L_{n}(i, k, t)}{d t} & =\delta L_{n}(i, k, t)+\lambda\left\{1-F_{n}\left[L_{n}(i, k, t)\right]\right\} L_{n}(i, k, t) \\
& -\lambda\left\{1-F_{n}\left[L_{n}(i, k+1, t)\right]\right\} L_{n}(i, k+1, t)+\lambda \int_{L_{n}(i, k+1, t)}^{L_{n}(i, k, t)} l f_{n}(l) d l
\end{aligned}
$$

and after partial integration we have

$$
\frac{d L_{n}(i, k, t)}{d t}=\delta L_{n}(i, k, t)+\lambda \int_{L_{n}(i, k+1, t)}^{L_{n}(i, k, t)}\left[1-F_{n}(l)\right] d l
$$

This recurrence differential equation determines the evolution of $L_{n}(i, k, t)$ through period $n$. The boundary conditions are obtained by taking the lefthand limit of $L_{n}(i, k, t)$ at $t=1$. Hereby we can distinguish the following three cases.

a) $k=K_{i}, K_{i}+1, \ldots$

According to the definition of characteristic claim number it follows from (4a) and (5) that

$$
\begin{array}{rlrl}
L_{n}(i, k, 1) & =w_{n+1} \sum_{j=1}^{J}\left[b_{n+1}(j)+A_{n+1}(j, 0,0)\right]\left[t_{i j}(k+1)-t_{i j}(k)\right] \\
& =0 & \text { for } k=K_{i}, K_{i}+1, \ldots
\end{array}
$$

and the solution of equation (6) reduces to

$$
L_{n}(i, k, t)=0 \quad \text { for } k=K_{i}, K_{i}+1, \ldots
$$

b) $n=N$

Using $(4 \mathrm{~b})$ and $(5)$ we find that

$$
L_{N}(i, k, 1)=A_{N}(i, k+1,1)-A_{N}(i, k, 1)=0
$$

so that the solution of $(6)$ is

$$
L_{N}(i, k, t)=\mathrm{o}
$$

The results (7) and (8) are intuitively appealing.

c) $k=0, \ldots, K_{i}-1$ and $n=1, \ldots, N-1$

According to $(4 \mathrm{a})$ a repeated use of (5) yields

$$
\begin{aligned}
L_{n}(i, k, 1) & =w_{n+1} \sum_{j=1}^{J}\left[b_{n+1}(j)+A_{n+1}(j, 0,0)\right]\left[t_{i j}(k+1)-t_{i j}(k)\right] \\
& =\ldots \\
& =w_{n+1} \sum_{j=1}^{J}\left[b_{n+1}(j)+A_{n+1}\left(j, K_{j}, 0\right)-L_{n+1}\left(j, K_{j}-1,0\right)\right. \\
& \left.-\ldots-L_{n+1}(j, 0,0)\right]\left[t_{i j}(k+1)-t_{i j}(k)\right]
\end{aligned}
$$


From (3) and (7) it follows that

$$
\frac{d A_{n+1}\left(j, K_{j}, t\right)}{d t}=\delta A_{n+1}\left(j, K_{j}, t\right)
$$

so that

$$
A_{n+1}\left(j, K_{j}, t\right)=e^{-\delta(1-t)} A_{n+1}\left(j, K_{j}, 1\right)
$$

and thus

$$
\begin{aligned}
A_{n+1}\left(j, K_{j}, 0\right) & =e^{-\delta} A_{n+1}\left(j, K_{j}, 1\right) \\
& =e^{-\delta} w_{n+2} \sum_{j=1}^{J}\left[b_{n+2}(j)+A_{n+2}(j, 0,0)\right] t_{i j}\left(K_{j}\right) \\
& =e^{-\delta} w_{n+2}\left[b_{n+2}(J)+A_{n+2}(J, 0,0)\right]
\end{aligned}
$$

This shows that $A_{n+1}\left(j, K_{j}, 0\right)$ is independent of $j$, so that we have for $k=0, \ldots, K_{i}-1$ and $n=1, \ldots, N-1$

(9) $\quad L_{n}(i, k, 1)=w_{n+1} \sum_{i=1}^{\prime}\left[b_{n+1}(j)-L_{n+1}\left(j, K_{j}-1,0\right)-\ldots-\right.$

$$
\left.-L_{n+1}(j, 0,0)\right]\left[t_{i j}(k+1)-t_{i j}(k)\right]
$$

In particular for $n=N-1$ this formula reduces to

$$
L_{N-1}(i, k, 1)=w_{N} \sum_{j=1}^{J} b_{N}(j)\left[t_{i j}(k+1)-t_{i j}(k)\right]
$$

5. THE OPTIMAL CRITICAL CLAIM SIZE IN THE CASE OF EXPONENTIALlY DISTRIBUTED CLAIM AMOUNTS

We assume that the claim amounts are distributed according to:

$F_{n}(l)=1-e^{-c_{n} l}$. Then equation (6) becomes

(10) $\frac{d L_{n}(i, k, t)}{d t}=\delta L_{n}(i, k, t)+\frac{\lambda}{c_{n}}\left[e^{-\boldsymbol{c}_{n} L_{n}(i, k+1, t)}-e^{-\boldsymbol{c}_{n} L_{n}(i, k, t)}\right]$

with given initial values $L_{n}(i, k, 1)$ for $k=0, \ldots, K_{i}-1$, and where $L_{n}(i, k, t)=0$ for $k=K_{i}, K_{i}+1, \ldots$

We make the substitution

$$
L_{n}(i, k, t)=\frac{1}{c_{n}} \ln \frac{\phi_{n}}{\phi_{n}(i, k+1, k)}
$$

where we put $\phi_{n}(i, k, t)=1$ for $k=K_{i}, K_{i}+1, \ldots$ 
Substitution in equation (10) leads to

$$
\begin{aligned}
& -\frac{1}{\phi_{n}(i, k, t)} \frac{d \phi_{n}(i, k, t)}{d t}+\lambda \frac{\phi_{n}(i, k+1, t)}{\phi_{n}(i, k, t)}-\delta \ln \phi_{n}(i, k, t) \\
& =\frac{1}{\phi_{n}(i, k+1, t)} \frac{d \phi_{n}(i, k+1, t)}{d t}+\lambda \frac{\phi_{n}(i, k+2, t)}{\phi_{n}(i, k+1, t)}-\delta \ln \phi_{n}(i, k+1, t) \\
& =\ldots \\
& =\lambda
\end{aligned}
$$

or

(12) $\frac{d \phi_{n}(i, k, t)}{d t}=\lambda \phi_{n}(i, k, t)-\lambda \phi_{n}(i, k+1, t)+\delta \phi_{n}(i, k, t) \ln \phi_{n}(i, k, t)$

For given $t$ we can compute the solutions $\phi_{n}(i, k, t), k=0, \ldots, K_{i}-1$, of (12) by successive approximations. We replace (12) by

$$
\left\{\begin{aligned}
\frac{d \phi_{n}^{(0)}(i, k, t)}{d t}=\lambda \phi_{n}^{(0)}(i, k, t)-\lambda \phi_{n}^{(0)}(i, k+1, t) & \\
\frac{d \phi_{n}^{(v)}(i, k, t)}{d t}=\left[\lambda+\delta \ln \phi_{n}^{(\nu-1)}(i, k, t)\right] \phi_{n}^{(v)}(i, k, t)-\lambda \phi_{n}^{(v)}(i, k+1, t) & v=1,2, \ldots
\end{aligned}\right.
$$

These equations are of the form

(14) $\frac{d \phi_{n}(i, k, t)}{d t}=(\lambda+a) \phi_{n}(i, k, t)-\lambda \phi_{n}(i, k+1, t)$

and have as solution

$$
\begin{aligned}
\phi_{n}(i, k, t)=\left(\frac{\lambda}{\lambda+a}\right)^{K_{i}-k}+e^{-(\lambda+a)(1-t)} & \sum_{l=k}^{K_{i}-1} \frac{[\lambda(1-t)]^{l-k}}{(l-k) !} \\
& {\left[\phi_{n}(i, l, 1)-\left(\frac{\lambda}{\lambda+a}\right)^{K_{i}-l}\right] }
\end{aligned}
$$

where

$$
\begin{aligned}
\phi_{n}(i, l, 1) & =e^{c_{n} L_{n}(i, l, 1)} \phi_{n}(i, l+1,1) \\
& =\cdots \\
& =\exp \left[c_{n} \sum_{k=l}^{K_{i}-1} L_{n}(i, k, 1)\right]
\end{aligned}
$$

The formulae (11), (15) and (16) determine the solution of equation (10). Together with (9) these formulae permit a policyholder to calculate his optimal critical claim size at each moment. 


\section{REFERENCES}

De Leve, G. and P. J. Weeda (1968). Driving with Markov-programming, Astin Bulletin, 5, 62-86.

GRENANDER, U. (1957). Some Remarks on Bonus Systems in Automobile Insurance, Skand. Aktuar.-Tidskr., 40, 180-197.

Haehling von Lanzenauer, C. (1974). Optimal Claim Decisions by Policyholders in Automobile Insurance with Merit-Rating Structures, Operations Research, 22, 979990. Reprinted in Actuarial Research Clearing House 1974.2.

Lemaire, J. (1976). Driver versus Company: Optimal Behaviour of the Policyholder, Scand. Actuarial J., 4, 209-219.

Lemaire, J. (1977). La Soif du Bonus, Astin Bulletin, 9, 181-19o.

Martin-Löf, A. (1973). A Method for Finding the Optimal Decision Rule for a Policy Holder of an Insurance with a Bonus System, Skand. Aktuar-Tidskr., 23-29.

Norberg, R. (1975). Credibility Premium Plans which Make Allowance for Bonus Hunger, Scand. Actuarial J., 3, 73-86.

Straub, E. (1969). Zur Theorie der Prämienstufensysteme, Mitteilungen der Verein. Schweizer. Versich. Math., 69, 75-85.

Weeda, P. J. (1975). Technical Aspects of the Iterative Solution of the Automobile Insurance Problem, Report of the Mathematical Centre BN27/75, Amsterdam. 